Journal of Mathematics and Statistics 3 (4): 181-187, 2007

ISSN 1549-3644

(C) 2007 Science Publications

\title{
Nonparametric Analysis on System Availability: Confidence Bound and Power Function
}

\author{
Jau-Chuan Ke and Yunn-Kuang Chu \\ Department of Statistics, National Taichung Institute of Technology, Taichung 404, Taiwan, R.O.C
}

\begin{abstract}
In this study we consider the steady-state availability, denoted $A$, of a system with distribution-free failure and repair time. In particular, we are interested in constructing a lower confidence bound and a testing framework for $A$. We first show that the natural estimator $\hat{A}$ of $A$, defined as the ratio of the failure time sample mean to the sum of the failure time sample mean and the repair time sample mean, is strongly consistent and asymptotically normal. Then using the asymptotic distribution of $\hat{A}$, we develop a lower confidence bound and a hypothesis test for $A$. Finally, a numerical simulation study is conducted in order to illustrate the performance of $\hat{A}$ in applied inference about $A$.
\end{abstract}

Key words: Availability, hypothesis test, lower confidence bound, power function, repairable system, simulation, Slutsky's theorem

\section{INTRODUCTION}

To assess the long-term performance of a repairable system, the steady-state availability of the system is often considered. Steady-state availability (henceforth availability) is defined as the ratio

$$
A=\frac{M T B F}{M T B F+M T T R},
$$

where $M T B F$ is the mean time between failures and MTTR represents the mean time to repair following failure. Availability is a very important measure in evaluating the long-term performance of a system, and numerous studies have sought to estimate efficient confidence intervals for the availability of repairable systems assuming various specified failure and repair time distributions. Confidence intervals for availability are given in Thompson ${ }^{[1]}$, Gary and Lewis ${ }^{[2]}$, Masters and Lewis ${ }^{[3]}$, Elperin and Gertsbakh ${ }^{[4]}, \mathrm{Mi}^{[5]}$, Masters et $a l .^{[6]}$, Chandrasekhar et al. ${ }^{[7]}$, Ananda ${ }^{[8]}$, Yadavalli et $a l . .^{[9]}$, Ananda ${ }^{[10]}$, Chandrsekhar ${ }^{[11]}$ and Lim et al. ${ }^{[12]}$, among others. But to the best of our knowledge there has been no research that explores statistical inference about availability in repairable systems with distribution-free failure and repair time.

The objective of this study was to present statistical inference for the availability $A$ of a system assuming distribution-free failure and repair time. In section 2 , we show that the natural estimator $\hat{A}$ of $A$ is strongly consistent and asymptotically normal. Using this result, we construct a $100(1-\alpha) \%$ lower confidence bound for $A$. Also, a test rule is established to test the null hypothesis $H_{0}: A \leq A_{0}$.

Statistical inference about availability: Let

$X$ represent the time between system failures and $Y$

Corresponding Author: Jau-Chuan Ke, Ph.D. Department of Statistics, National Taichung Institute of Technology, No. 129, Sec. 3, Sanmin Rd.,Taichung 404, Taiwan, R.O.C. 
denote the time to repair the system. Then the system availability is defined as

$$
A=\frac{\mu_{X}}{\mu_{X}+\mu_{Y}}
$$

where $\mu_{X}$ is the mean time between failures and $\mu_{Y}$ is the mean time to repair.

\section{Estimating availability: Assume that} $X_{1}, X_{2}, \cdots, X_{n}$ is a random sample of failure times and $Y_{1}, Y_{2}, \cdots, Y_{n}$ is a random sample of repair times. Let $\bar{X}$ and $\bar{Y}$ represent the sample means of the $X \mathrm{~s}$ and $Y \mathrm{~s}$, respectively. According to the strong law of large numbers ${ }^{[13]}, \quad \bar{X}$ is a strongly consistent estimator of $\mu_{X}$ and $\bar{Y}$ is a strongly consistent estimator of $\mu_{Y}$. Hence a strongly consistent estimator of the availability is

$$
\hat{A}=\frac{\bar{X}}{\bar{X}+\bar{Y}}
$$

In practical systems, the distributions of $X$ and $Y$ are seldom known, so the exact distribution of $\hat{A}$ cannot be derived. Nevertheless, the asymptotic distribution of $\hat{A}$ is obtained as follows.

First, according to the central limit theorem ${ }^{[14]}$, we have

$$
\sqrt{n}\left(\bar{X}-\mu_{X}\right) \stackrel{D}{\longrightarrow} N\left(0, \sigma_{X}^{2}\right)
$$

and

$$
\sqrt{n}\left(\bar{Y}-\mu_{Y}\right) \stackrel{D}{\longrightarrow} N\left(0, \sigma_{Y}^{2}\right)
$$

where $\sigma_{X}^{2}$ and $\sigma_{Y}^{2}$ are the variances of $X$ and $Y$, respectively, and $\stackrel{D}{\longrightarrow}$ denotes convergence in distribution. Next note that

$$
\begin{aligned}
& \sqrt{n}(\hat{A}-A)=\sqrt{n}\left(\frac{\bar{X}}{\bar{X}+\bar{Y}}-\frac{\mu_{X}}{\mu_{X}+\mu_{Y}}\right) \\
& =\frac{\sqrt{n}\left[\mu_{Y}\left(\bar{X}-\mu_{X}\right)-\mu_{X}\left(\bar{Y}-\mu_{Y}\right)\right]}{\left(\mu_{X}+\mu_{Y}\right)(\bar{X}+\bar{Y})}
\end{aligned}
$$

Therefore, we obtain

$$
\sqrt{n}(\hat{A}-A) \stackrel{D}{\longrightarrow} N\left(0, \sigma^{2}\right)
$$

where

$$
\sigma^{2}=\left(\mu_{X}^{2} \sigma_{Y}^{2}+\mu_{Y}^{2} \sigma_{X}^{2}\right) /\left(\mu_{X}+\mu_{Y}\right)^{4} \text {. }
$$

Now set

$\hat{\sigma}^{2}=\left(\bar{X}^{2} S_{Y}^{2}+\bar{Y}^{2} S_{X}^{2}\right) /(\bar{X}+\bar{Y})^{4}$

where

$$
S_{X}^{2}=\frac{1}{n} \sum_{i=1}^{n}\left(X_{i}-\bar{X}\right)^{2} \quad \text { and } \quad S_{Y}^{2}=\frac{1}{n} \sum_{i=1}^{n}\left(Y_{i}-\bar{Y}\right)^{2} .
$$

Then $\hat{\sigma}^{2}$ is a strongly consistent estimator of $\sigma^{2}$. Applying Slutsky's theorem ${ }^{[14]}$, we deduce that

$$
\sqrt{n}(\hat{A}-A) / \hat{\sigma} \stackrel{D}{\longrightarrow} N(0,1)
$$

Thus $\hat{A}$ is a (strongly) consistent and asymptotically normal (CAN) estimator with approximate variance $\hat{\sigma}^{2} / n$.

A Lower Confidence Bound:Using the CAN estimator $\hat{A}$ and its associated approximate variance $\hat{\sigma}^{2} / n$, we construct a lower confidence bound for the availability of a system with distribution-free failure and repair time. Let $z_{\alpha}$ be the upper $\alpha$ th quantile of the standard normal distribution; by the asymptotic distribution of $\sqrt{n}(\hat{A}-A) / \hat{\sigma}$ in expression (8), an approximate $100(1-\alpha) \%$ lower confidence bound of $A$ is obtained as

$$
\begin{aligned}
& 1-\alpha \approx P\left(\sqrt{n}(\hat{A}-A) / \hat{\sigma} \geq-z_{\alpha}\right) \\
& =P\left(\sqrt{n}(\hat{A}-A) / \hat{\sigma} \leq z_{\alpha}\right) \\
& =P\left(\hat{A}-z_{\alpha} \frac{\hat{\sigma}}{\sqrt{n}} \leq A\right) .
\end{aligned}
$$

Consequently, an approximate $100(1-\alpha) \%$ lower confidence bound is

$$
L C B=\hat{A}-z_{\alpha} \frac{\hat{\sigma}}{\sqrt{n}}
$$


A hypothesis test: In addition to the point and interval estimation, we are also interested in statistical testing about the availability for a system with distribution-free failure and repair time. Suppose that we want to test the hypothesis

$H_{0}: A \leq A_{0}$ versus $H_{a}: A>A_{0}$,

where $A_{0}$ is a constant level of availability.

If $A$ is viewed as a population mean, then the above testing problem is similar to that of testing a normal population mean. The CAN estimator $\hat{A}$ leads to a natural test statistic in this context, and the condition for rejecting $H_{0}$ is

reject $H_{0}$ if $\hat{A}>C_{\alpha}$,

where $\alpha$ is the pre-specified significance level of the test. And $C_{\alpha}$ is the critical value satisfying

$\alpha=P\left(\hat{A}>C_{\alpha} \mid A=A_{0}\right)$

$=P\left(\frac{\sqrt{n}\left(\hat{A}-A_{0}\right)}{\hat{\sigma}}>\frac{\sqrt{n}\left(C_{\alpha}-A_{0}\right)}{\hat{\sigma}} \mid A=A_{0}\right)$.

Since $\sqrt{n}\left(\hat{A}-A_{0}\right) / \hat{\sigma} \stackrel{D}{\longrightarrow} N(0,1), \quad C_{\alpha} \quad$ can be determined by $\sqrt{n}\left(C_{\alpha}-A_{0}\right) / \hat{\sigma}=z_{\alpha}$, that is

$C_{\alpha}=A_{0}+z_{\alpha} \frac{\hat{\sigma}}{\sqrt{n}}$

Using the above rejection rule (10), the power function is, for $A \geq A_{0}$, given by

$$
\begin{aligned}
& \beta_{n}(A)=P\left(\hat{A}>C_{\alpha} \mid A\right) \\
& =P\left(\frac{\sqrt{n}(\hat{A}-A)}{\hat{\sigma}}>\frac{\sqrt{n}\left(C_{\alpha}-A_{0}\right)}{\hat{\sigma}} \mid A\right) \\
& =P\left(\frac{\sqrt{n}(\hat{A}-A)}{\hat{\sigma}}>z_{\alpha}-\frac{\sqrt{n}\left(A-A_{0}\right)}{\hat{\sigma}} \mid A\right)
\end{aligned}
$$

Based upon result (8), $\beta_{n}(A)$ can be estimated by
$\hat{\beta}_{n}(A)=1-\Phi\left(z_{\alpha}-\frac{\sqrt{n}\left(A-A_{0}\right)}{\hat{\sigma}}\right)$

where $\Phi$ is the cumulative standard normal distribution function.

In summary, we use the asymptotic distribution of $\hat{A}$ described in (8) to determine the rejection rule and the approximate power of the test.

Simulation study: In order to explore the performance of $\hat{A}$, we conduct a three-part simulation study. First, the accuracy of the lower confidence bound $L C B$ of $A$ is evaluated using its coverage percentage. Second, the type I error rate (i.e., the significance level of the test) is simulated with three different failure time distributions. Third, the power functions of the test under different levels of $n$ are generated and compared. For concreteness, we consider a Weibull failure time distribution with shape and scale parameters $b$ and $a$, respectively. Note that the failure-rate parameter of Weibull distribution is increasing (IFR) for $b>1$, decreasing (DFR) for $0<b<1$ and constant (CFR) for $b=1^{[15]}$. For convenience, the repair time distribution is assumed to be exponential with unit mean.

Simulating the lower confidence bound: We simulate $L C B=\hat{A}-z_{\alpha} \hat{\sigma} / \sqrt{n}$ as follows. The values of $b$ are set to $0.5,1$, and 2 so that the failure rate is decreasing $(D F R)$, constant $(C F R)$, and increasing (IFR), respectively. For a given value of $b$, the value of $a$ is chosen in such a way that $\mu_{X} / \mu_{Y}=10 / 1=10$ so that $A=\mu_{X} /\left(\mu_{X}+\mu_{Y}\right)=0.9091$.

For each combination of $b$ and $a$, random samples of failure times $\left(x_{1}, x_{2}, \cdots, x_{n}\right)$ and repair times $\left(y_{1}, y_{2}, \cdots, y_{n}\right)$ are drawn from $X$ and $Y$, 
respectively. Then the estimate $\hat{A}$ and its associated variance $\hat{\sigma}^{2} / n$ are computed. The $95 \% L C B$ of $A=$ 0.9091 is given as

$$
L C B=\hat{A}-z_{0.05} \hat{\sigma} / \sqrt{n}=\hat{A}-1.645 \hat{\sigma} / \sqrt{n}
$$

The simulation is replicated $N=1000$ times and we record the fraction of times that the $95 \% L C B$ is smaller than the true availability $A=0.9091$, which is called the coverage percentage. This process is repeated for $n=10,20, \cdots, 1000$. All simulations are performed on a PC Pentium IV using the software MATLAB ${ }^{\circledR}$ 7.0.4.

Since the number of $L C B \mathrm{~s}$ less than the true availability $A=0.9091$ follows a binomial distribution with $N=1000$ and $p=0.95$, the $99 \%$ confidence interval for the coverage percentage itself is

$0.95 \pm 2.576 \sqrt{0.95(1-0.95) / 1000}=0.95 \pm 0.0178$

or $(0.9322,0.9678)$. The simulation results for the performance of $\hat{A}$ in terms of the $L C B$ are presented in Fig. 1. For each kind of failure rate $(D F R, C F R$, and IFR), the corresponding 95\% LCB coverage percentages are plotted versus $n$ from 10 to 1000. All three curves almost appear in the $99 \%$ confidence interval $(0.9322,0.9678)$ and fluctuate along the nominated 95\% level provided that $n$ reaches large enough ( $n \geq 100$ ). Evaluating the chance of the three curves once inside the $99 \%$ confidence interval, it appears that ordering these curves by their relative performance on coverage fraction produces $D F R>C F R>I F R$.

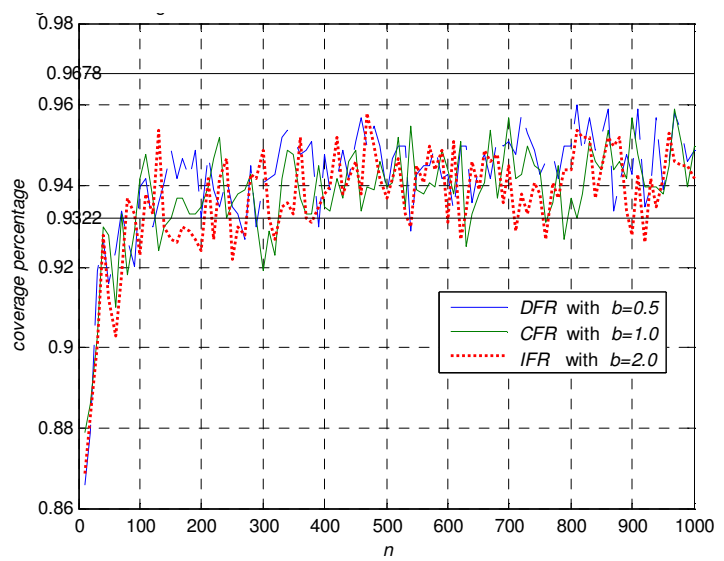

Fig. 1: Coverage fraction of $95 \%$ lower confidence bound for different values of $n$ and $b$

Simulating the hypothesis test: In this subsection, we explore the performance of the rejection rule given in (10) corresponding to the competing hypotheses

$$
H_{0}: A \leq A_{0} \text { versus } H_{a}: A>A_{0} .
$$

As before, the values of $b$ are set to $0.5,1$, and 2 so that the failure rates are decreasing, constant, and increasing, respectively. For a given value of $b$, the value of $a$ is selected such that $A=A_{0}=0.90$. We again set the significance level of the test to be $\alpha=0.05$. For each combination of $b$ and $a$, a sequence of estimates and variances are generated and denoted $\left(\hat{A}_{1}, \hat{\sigma}_{1}^{2} / n\right)$, $\left(\hat{A}_{2}, \hat{\sigma}_{2}^{2} / n\right), \cdots,\left(\hat{A}_{1000}, \hat{\sigma}_{1000}^{2} / n\right)$. Define $I_{j}$ as the indicator of the rejection event $\left\{\sqrt{n}\left(\hat{A}_{j}-A_{0}\right) / \hat{\sigma}_{j}>z_{0.05}\right\} \quad$ for $\quad j=1,2, \cdots, 1000$. Then $\hat{\alpha}_{n}=\sum_{j=1}^{1000} I_{j} / 1000$ is a simulated value of the predetermined type I error rate $\alpha=0.05$. We calculate $\hat{\alpha}_{n}$ for $n=10,20, \cdots, 1000$ by repeating 
this process. The simulation results for the type I error rate are illustrated in Fig. 2.

Like the $L C B$, a $99 \%$ confidence interval for the type I error rate is constructed as

$0.05 \pm 2.576 \sqrt{0.05(1-0.05) / 1000}=0.05 \pm 0.0178$ (16)

The goodness of performance for the simulated type I error rate scrutinized by the chance of three curves once inside the $99 \%$ confidence interval $(0.0322$, 0.0678) is summarized in Fig. 2. We find that all the three curves are almost contained within the 99\% confidence interval and fluctuate along the nominal level $5 \%$ when $n$ reaches sufficiently large $(n \geq 100)$. Based on this criterion, one can see that the DFR curve performs best, and the $C F R$ curve outperforms the IFR curve.

In addition to using $\hat{A}$ to construct the $L C B$ of $A$ and compute the type I error rate of the hypothesis test, we also use simulations to scrutinize its performance in terms of the power function of the hypothesis test. According to formula (13), we compute

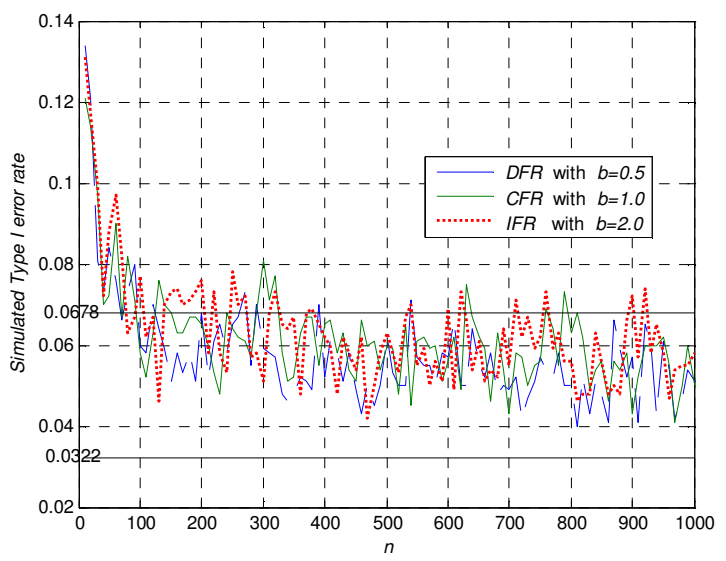

Fig. 2: Simulated type 1 error rate for different values aof $n$ and $b\left(\mathrm{~A}_{o}=0.90\right)$ $\hat{\beta}_{n j}(A)=1-\Phi\left(\left(1.645-\frac{\sqrt{n}\left(A-A_{0}\right)}{\hat{\sigma}_{j}}\right)\right.$

for $j=1,2, \cdots, 1000$. Then $\quad \widetilde{\beta}_{n}(A)=\sum_{j=1}^{1000} \hat{\beta}_{n j}(A) / 1000$

is a simulated power function at significance level $\alpha=0.05$. We construct the simulated power function $\widetilde{\beta}_{n}$ at $n=10,30,50,100$. It follows that for each kind of failure time distribution there are four simulated power curves. Simulation results for the power function are shown in Fig. 3, 4 and 5 corresponding to $b=0.5$ (DFR), $b=1.0(C F R)$, and $b=2.0(I F R)$, respectively. We find that $\widetilde{\beta}_{100}(A)>\widetilde{\beta}_{50}(A)>\widetilde{\beta}_{30}(A)>\widetilde{\beta}_{10}(A)$ for $A \geq A_{0}$, all curves approach 1 as $n$ large enough. Figures 6 and 7 indicate that $\widetilde{\beta}_{n}(I F R)>\widetilde{\beta}_{n}(C F R)>\widetilde{\beta}_{n}(D F R)$ for each fixed $n$. In other words, the results suggest that $\widetilde{\beta}_{n_{1}}>\widetilde{\beta}_{n_{2}}$ for $n_{1}>n_{2}$, and the power increases

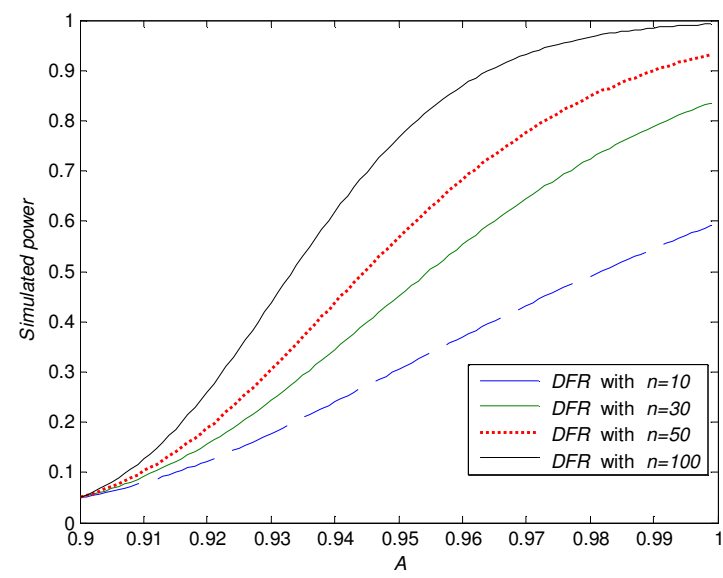

Fig. 3: Simulated curves of power function for different values of $n\left(\mathrm{~A}_{o}=0.90\right)$ 


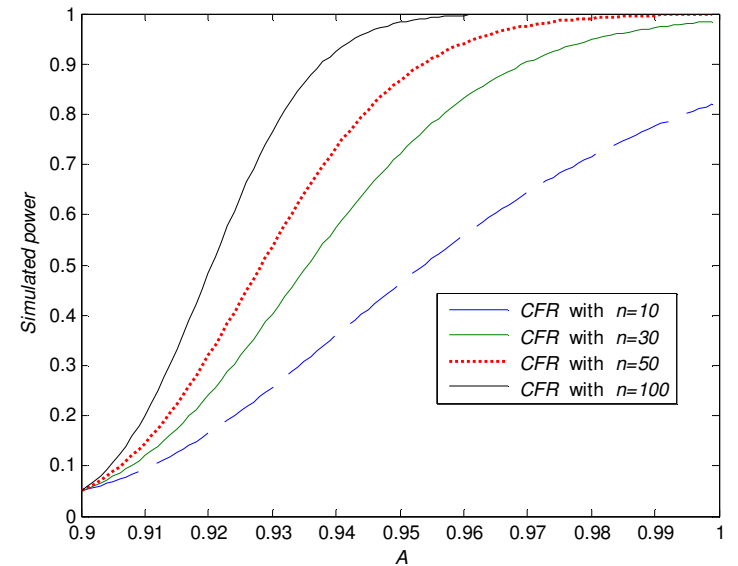

Fig. 4: Simulated curves of power function for different values of $n\left(\mathrm{~A}_{o}=0.90\right)$

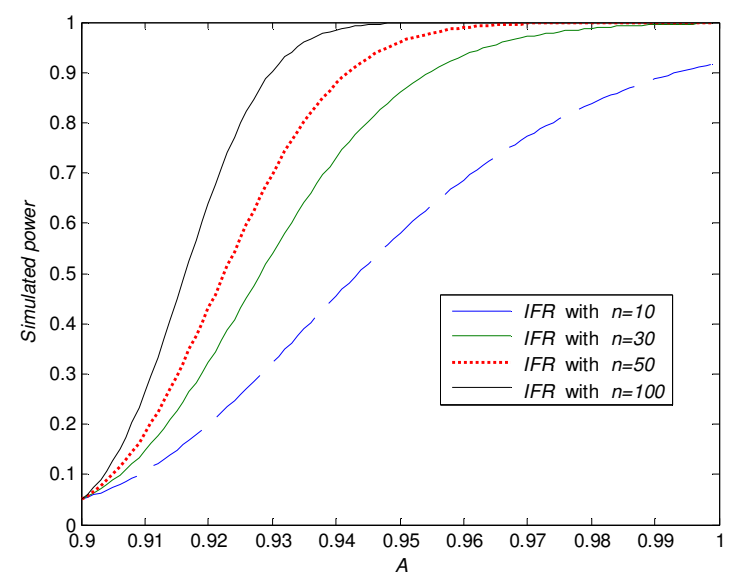

Fig. 5: Simulated curves of power function for different values of $n\left(\mathrm{~A}_{o}=0.90\right)$

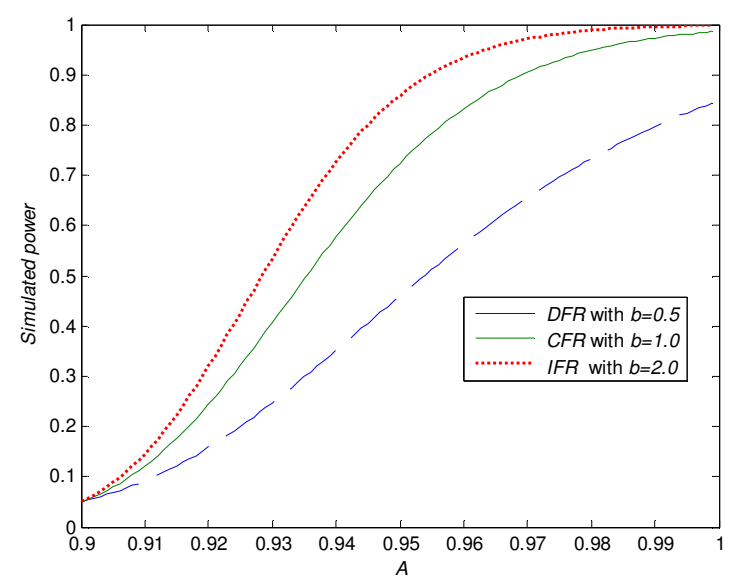

Fig. 6: Simulated curves of power function for different values of $\mathrm{b}\left(n=30\right.$ and $\left.A_{0^{-}} 0.90\right)$

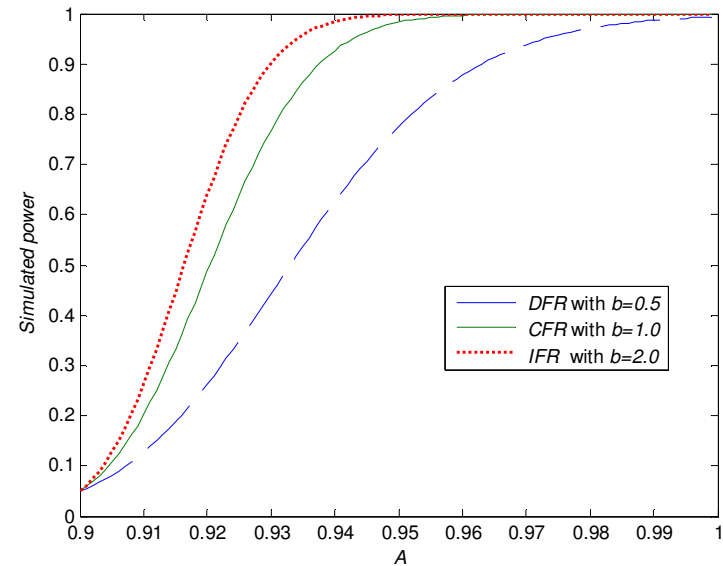

Fig. 7: Simulated curves of power function for different values of $b\left(n=100\right.$ and $\left.A_{0^{-}}-0.90\right)$

with the failure rate (shape parameter $b$ ). It is not surprising that when all parameters are given, $\widetilde{\beta}_{n}$ quickly approaches maximum power as $n$ increases. Based on these numerical investigations, we conclude that $\hat{A}$ and its estimated variance $\hat{\sigma}^{2} / n$ appear to perform well in power calculations provided $n$ or the shape parameter $(b)$ is sufficiently large.

\section{DISCUSSION AND CONCLUSION}

This study provides a statistical inference for the availability $A$ of a repairable system with distribution-free failure and repair time. We show that the natural estimator $\hat{A}$ is strongly consistent and asymptotically normal with approximate variance $\hat{\sigma}^{2} / n$. Using the asymptotic results, we construct a lower confidence bound $(L C B)$ and develop a hypothesis test framework for $A$. Through the numerical simulation study, we find the CAN estimator appears to perform well on interval estimation and hypothesis testing. Specifically, considering three types of failure rates-decreasing $(D F R)$, constant $(C F R)$, and increasing $(I F R)$ - we conclude the following.

* The $L C B$ of $A, \hat{A}-Z_{\alpha} \hat{\sigma} / \sqrt{n}$, performs well if $n$ large enough $(n \geq 100)$.

* The $L C B$ corresponding to DFR outperforms that corresponding to $I F R$, based on coverage fraction.

* The type I error rate of the test $H_{0}: A \leq A_{0}$ versus $H_{a}: A>A_{0}$ is close to the nominal level when $n$ is sufficiently large $(n \geq 100)$. 
* The type I error rate corresponding to $D F R$ outperforms that corresponding to IFR, based on the chance of the corresponding curve once inside its $99 \%$ confidence interval.

* The power of the test $H_{0}: A \leq A_{0}$ versus $H_{a}: A>A_{0}$ increases with $n$.

* The rejection region $\left\{\hat{A}>C_{\alpha}\right\}$ is unbiased (i.e., $\beta_{n}(A)>\beta_{n}\left(A_{0}\right)$ for $\left.A>A_{0}\right)$.

* The power quickly reaches the maximum 1 when $n$ increases.

* For a fixed sample size $n$, the power function corresponding to IFR exceeds that corresponding to $D F R$. That is, the greater the shape parameter $(b)$, the better the power function performs.

We strongly believe that the statistical inference technique proposed by this paper can be successfully, efficiently, and easily applied to real systems. Future research may consider generalizing the asymptotic estimator to a parallel system or comparing availability in two repairable systems.

\section{REFERENCES}

1. Thompson, M., 1966. Lower confidence limits and a test of hypothesis for system availability. IEEE Transactions on Reliability, 15: 32-36.

2. Gary, H.L. and T.O. Lewis, 1967. A confidence interval for the availability ratio. Technometrics, 9: 465-471.

3. Masters, B.N. and T.O. Lewis, 1987. A note on the confidence interval for the availability ratio. Microelectronics \& Reliability, 27: 487-492.

4. Elperin, T. and I. Gertsbakh, 1988. Lower confidence limit for the availability of a parallel system with renewable components. Communications in Statistics, Theory and Methods, 17: 311-323.

5. Mi, J., 1991. Interval estimation of availability of a series system. IEEE Transactions on Reliability, 40: 541-546.
6. Masters, B.N., T.O. Lewis and W.J. Kolarik, 1992. A confidence interval for the availability ratio for systems with Weibull operating time and lognormal repair time. Microelectronics \& Reliability, 32: 89-99.

7. Chandrasekhar, P., P. Natrajan and H.S. Sujatha, 1994. Confidence limits for the steady state availability of systems. Microelectronics \& Reliability, 34: 1365-1367.

8. Ananda, M.M.A., 1999. Estimation and testing of availability of a parallel system with exponential failure and repair times. J. Stat. Planning and Inference, 77: 237-246.

9. Yadavall, V.S.S., M. Botha and A. Bedder, 2002. Asymptotic confidence limits for the steady state availability of a two-unit parallel system with preparation time for the repair facility. Asia-Pacific J. Operational Res., 19: 249-256.

10. Ananda, M.M.A., 2003. Confidence intervals for steady state availability of a system with exponential operating time and lognormal repair time. Appl. Math. and Comp., 137: 499-509.

11. Chandrasekjar, P., R. Natarajan and V.S.S. Yadavalli, 2004. A study on a two unit standby system with Erlangian repair time. Asia-Pacific J. Operational Res., 21: 271-277.

12. Lim, J.H., S.W. Shin, D.K. Kim and D.H. Park, 2004. Bootstrap confidence intervals for steady-state availability. Asia-Pacific J. Operational Res., 21: 407-419.

13. Roussas, G.G., 1997. A Course in Mathematical Statistics. ${ }^{\text {nd }}$ Edn., Academic Press.

14. Hogg, R.V. and A.T. Craig, 1995. Introduction to Mathematical Statistics. Prentice-Hall, Inc.

15. Saunders S.C., 2006. Reliability, Life Testing and the Prediction of Service Lives: for Engineers and Scientists, Springer. 